\title{
AUTOMORPHISMS OF FINITE GROUPS AND THEIR FIXED-POINT GROUPS
}

\author{
J. N. WARD
}

(Received 3 October 1967; revised 20 February 1968)

\section{Introduction}

Let $G$ denote a finite group with a fixed-point-free automorphism of prime order $p$. Then it is known (see [3] and [8]) that $G$ is nilpotent of class bounded by an integer $k(p)$. From this it follows that the length of the derived series of $G$ is also bounded. Let $l(p)$ denote the least upper bound of the length of the derived series of a group with a fixed-point-free automorphism of order $p$. The results to be proved here may now be stated:

THEOREM 1. Let $G$ denote a soluble group of finite order and $A$ an abelian group of automorphisms of $G$. Suppose that

(a) $|G|$ is relatively prime to $|A|$;

(b) $G_{A}$ is nilpotent and normal in $G_{\omega}$ for all $\omega \in A^{\not \# \text {; }}$

(c) the Sylow 2-subgroup of $G$ is abelian; and

(d) if $q$ is a prime number and $q^{k}+1$ divides the exponent of $A$ for some integer $k$ then the Sylow q-subgroup of $G$ is abelian.

Then if $G_{\omega}^{(n)}=1$ for all $\omega \in A^{\#}, G_{A}^{(m)}=1$ and $p$ is any prime divisor of $|A|$, it follows that $G^{(t)}$ is nilpotent where $t=\max (n, 1(p)+m-1)$.

THEOREM 2. Let $G$ be a group of order prime to $p$ with a group of automorphisms $A$ of order $p^{2}$ and exponent $p$. Suppose that for each $\omega \in A^{\#}$, $G_{\omega}$ is nilpotent. Then $G / F(G)$ belongs to the variety $V$ generated by all groups. with a fixed-point-free automorphism of order $p$.

Notation. The notation is standard and agrees with that in [9] with the following additions. If $G$ is a finite group then $|G|$ denotes the order of $G$; $G^{\#}$ denotes the set of non-identity elements of $G$; $G^{(n)}$, where $n$ is a positive integer, denotes the $n$-th derived group of $G$; and $F(G)$ denotes the Fitting subgroup of $G$.

If $A$ denotes a group of operators acting on $G$ then $G_{\omega}$, for $\omega \in A$, denotes the subgroup of $G$ consisting of those elements fixed by $\omega . G_{A}$ denotes the subgroup of $G$ consisting of all those elements of $G$ left fixed by all the operators in $A$. 
If $H$ and $K$ are groups then $H$ wr $K$ denotes the wreath product of $H$ with $K$. Thus $H$ wr $K$ contains a normal subgroup $N$ which is a direct product of $|K|$ copies of $H$ and a complementary subgroup isomorphic to $K$.

Discussion OF THE RESULTS. In order to illustrate Theorem 1 the special case in which $|A|=2$ may be mentioned. In this case the conditions (c) and (d) are automatically satisfied and the theorem reduces to the first part of [9] Theorem 1. Indeed, subject to the restrictions imposed by the conditions (c) and (d), the theorem of H. Kurzweil [4] and Theorem 1 generalise the Kovács-Wall theorem [6] and [9] Theorem 1 to the case in which the automorphism group is abelian.

Another consequence of theorem 1 is:

COROLLARY. Let $G$ be a soluble group with a fixed-point-free automorphism $\omega$ of order $p^{2}$, where $p$ is some prime. If the Sylow 2-subgroup of $G$ is abelian then $G^{(2 l(p)-1)}$ is nilpotent.

This corollary contains the essential part of theorem 2 in [10] (although a stronger result in this case is proved in [5]). The corollary is obtained by applying theorem 1 to the group $G$ and the automorphism group generated by $\omega^{p}$.

The hypotheses (c) and (d) arise from applications of [2] Theorem B and [7] Theorem 3.1 respectively. Although the restrictions are necessary in these theorems, it is not known whether they are needed for the truth of Theorem 1.

Since we are assuming in Theorem 1 that the Sylow 2-subgroup of $G$ is abelian, we can let $l(p)$ be the least upper bound of the length of the derived series of a group of odd order with a fixed-point-free automorphism of order $p$. This change will not alter the validity of the theorem or its proof. Subject to this alteration we will display examples to show that in certain respects Theorem $\mathbf{l}$ is the best possible. The point of each example is given below but the actual constructions are deferred until section 5 of the paper.

In example 1 we exhibit a group $G$ with an automorphism group $A$ of prime order satisfying all the conditions of the theorem. The point of this example is to show that $G^{(t)}$, where $t$ is defined as in Theorem 1 , is the first nilpotent term in the derived series of $G$.

Whenever the automorphism group $A$ in Theorem 1 is cyclic of prime order then we have $m=n$ and $t=l(p)+m-1$. Groups of the type exhibited in example 1 show that in this case $G^{(n)}$ need not be nilpotent. In example 2 we display groups for which $m$ is less than $n$ and $G^{(l(p)+m-1)}$ is not nilpotent. For these groups we have $t=n$.

The groups constructed in [9], page 480 , show that the assumption 
that $G_{A}$ is nilpotent is necessary, even when the remainder of hypothesis (b) and all the other assumptions in Theorem 1 are satisfied. In example 3 we will give an example to show that the other assumption given in (b) is also necessary, even when the other hypotheses are satisfied.

Turning now to Theorem 2, we mention that using the methods described in section 5, it is possible to show the following: let $Q$ be a group with a fixed-point-free automorphism of order $p$; then there exists a group $G$ satisfying the hypothesis of theorem 2 and for which we have $G / F(G) \cong Q$.

I thank the referee for his comments on an earlier form of this paper.

\section{Preliminary Lemmas}

The following result of $\mathrm{H}$. Kurzweil is obtained by combining [4] lemma 5 and [7] Theorem 4.1.

Lemma 1. Let $A$ be an abelian group of operators on the soluble group $G$. Assume that $A$ and $G$ satisfy the conditions (a) and (d) of Theorem 1. Let $G A$ denote the split extension of $G$ by $A$.

Suppose that $V$ is a vector space over a field $\mathscr{F}$ of characteristic $q$ where $q$ is not a divisor of $|A|$. Assume that $V$ yields a representation of $G A$ whose restriction to $G$ is homogeneous and faithful. Also assume that every $q^{\prime}$-subgroup of $G_{\omega}$ for each $\omega \in A^{\#}$ centralises $V_{A}$.

Then there exists $\phi \in A^{\# \# ~ s u c h ~ t h a t ~} G_{\phi}=G$.

The next lemma follows from [1], section 248, (although the 'theorem' stated at the end of that section is talse).

Lemma 2. Let $A$ be an abelian group of operators on the group $G$. Suppose that each element of $A^{\#}$ acts fixed-point-free on $G$. Then $A$ is cyclic.

\section{Proof of Theorem 1}

The theorem is to be proved by induction on the group order and by way of contradiction. Suppose that $G$ and $A$ form a counterexample to the theorem, with the order of $G$ as small as possible. Let $G A$ be the split extension of $G$ by $A$.

If $H / K$ is an $A$-factor of $G$ then either $A$ is represented faithfully on

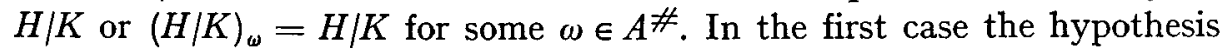
of the theorem is satisfied by $H / K$ so, provided that $H / K \neq G / 1$, we know that $H / K$ satisfies the conclusion by induction. In the second case, since the orders of $G$ and $A$ are relatively prime, $H / K$ is isomorphic to a section of $G_{\omega}$ and therefore satisfies the conclusion of the theorem. Thus by [6], lemma $2, F(G)$ is the unique minimal normal $A$-subgroup of $G$. Let $F=F(G)$. 
If $\omega$ is a non-trivial element of $A$ then $(G / F)_{\omega} \neq G / F$ or else $G^{(t)}$ is contained in $F$ which is contrary to the definition of $G$. Now if $F_{\omega}=F$ for some $\omega \in A^{\#}$ then $(G / F)_{\omega}=G / F$, since the solubility of $G$ implies that $C_{G}(F) \leqq F$. This contradiction shows that $F_{\omega} \neq F$ and hence that $A$ is represented faithfully on $F$.

Since $F$ is the unique minimal normal $A$-subgroup of $G, F$ is elementary abelian. Thus $F$ may be considered to be a $G A / F$ module over the appropriate Galois field. Since $F$ is irreducible as a $G A / F$ module and $G / F$ is normal in $G A / F$, we have by Clifford's theory:

$$
F=F_{1} \oplus F_{2} \oplus \cdots \oplus F_{u}
$$

where the $F_{i}$ are the homogeneous $G / F$-components of $F$. The group $A$ permutes these components transitively. Let $\bar{A}$ dencte the subgroup

$$
\left\{\omega \in A \mid F_{i}^{\omega}=F_{i}\right\}
$$

of $A$. Since $A$ is abelian and transitive on the $F_{i}, \bar{A}$ is independent of $i$.

If $\omega \in A^{\#}$ and $R$ is a Sylow subgroup of $G_{\omega}$ of order prime to the order of $F$ then $R$ normalises $F_{A}$. By [4], lemma 1 (iii) if $U$ is a subgroup of $G_{A}$ then

In particular we have

$$
N_{G}(U)=C_{G}(U)\left(N_{G}(U)\right)_{A} .
$$

$$
N_{G}\left(F_{A}\right)=C_{G}\left(F_{A}\right)\left(N_{G}\left(F_{A}\right)\right)_{A} .
$$

Since $G_{A}$ is nilpotent and the order of $R$ is prime to the order of $F_{A}$, it follows that $R \leqq C_{G}\left(F_{A}\right)$. From this we can conclude that for each $i$ $(i=1,2, \cdots, u), R \leqq C_{G}\left(\left(F_{i}\right)_{A}\right)$.

Suppose that $\bar{A} \neq 1$. Let $K_{i}=C_{G}\left(F_{i}\right)$. Now for each value of $i$, the group of operators $A$ on the group $G / K_{i}$ and the vector space $F_{i}$ satisfy the hypotheses of lemma 1. Thus there exist elements $\omega_{1}, \omega_{2}, \cdots, \omega_{u}$ in $A^{\#}$ such that for each $i,\left(G / K_{i}\right)_{\omega_{i}}=G / K_{i}$. Hence $G / K_{i}$ is isomorphic to a section of $G_{\omega_{i}}$ and we can conclude that $G^{(t)} \leqq K_{i}$ for each $i$. It now follows that

$$
G^{(t)} \leqq \bigcap_{i=1}^{u} K_{i}=C_{G}(F)=F .
$$

Since this is contrary to the definition of $G$ we must have $\bar{A}=1$.

Now let $v \in F_{i}$ and set $w=\sum_{\omega \in A} v \omega$. Then $w \in F_{A}$ so that if $x \in G_{\omega}$ (for some $\omega \in A^{\# \text { ) }}$ ) is of order prime to $|F|$ then $w x=w$. Equating the $F_{i}$ compcnents of each side of this equation we find that $v x=v$. Since $v$ was an arbitrary element of $F_{i}$ we may conclude that $x$ centralises $F_{i}$. But this applies for all possible values of $i$ so that we have $x \in C_{G}(F)=F$. Since $x$ was of order prime to the order of $F$ it follows that $x=1$. Thus we have shown for each $\omega \in A^{\#}$ that $G_{\omega}$ is a group of prime power order. 
Suppose that $F$ is a $q$-group. Then $F_{2}(G) / F$ is a $q^{\prime}$-group. Since $F_{A}>1$,

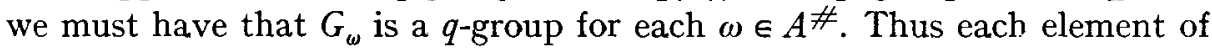
$A^{\#}$ acts fixed-point-free on $F_{2}(G) / F$ and, by lemma $2, A$ is cyclic. Let $\omega$ denote a generator of $A$; now $G_{A}=G_{\omega}$.

If $q=2$ then since the Sylow 2-subgroup of $G$ is abelian and $C_{G}(F)=F, F$ must be the Sylow 2-subgroup of $G$. Now if $\omega^{r} \neq 1$ then since $G_{\omega^{r}}$ is a 2-group, we have $(G / F)_{\omega^{r}}=1$. Thus $G / F$ is nilpotent of derived length not greater than $l(p)$ where $p$ is any prime dividing the order of $\omega$. Since this is contrary to the definition of $G$ we may suppose that $q \neq 2$.

Now suppose that $\phi \in A$ and that $G_{\phi}$ has derived length $k$. We will show that $G_{\phi}^{(k-1)} \leqq F$. Since $F_{A} \leqq G_{\phi}$, we have $\left(F_{A}, G_{\phi}^{(k-1)}\right) \leqq G_{\phi}^{(k-1)}$. Therefore $\left(\left(F_{A}, G_{\phi}^{(k-1)}\right), G_{\phi}^{(k-1)}\right)=1$. In the additive nctation this implies that if $v \in F_{A}$ and $x \in G^{(k-1)}$ then $v(1-x)^{2}=0$. Now if $w \in F_{i}$ for some $i$ then $v=\sum_{\theta \in A} w \theta \in F_{A}$. Thus for this $v$ we have $v(1-x)^{2}=0$. Equating the $F_{i}$ compcnents of each side of this relation we find that $w(1-x)^{2}=0$. Since $w$ was an arbitrary element of $F_{i}, i$ was arbitrary and $F$ is the sum of the $F_{i}$ it follews that for all $v \in F$ we have $v(1-x)^{2}=0$. But since $q$ is odd and the Sylow 2-subgroup of $G$ is abelian, if $x F$ has order $q^{r}$ in $G / F$ then ([2], theorem B) the minimal equation of $x F$ on $F$ is of order $q^{r}$. Now if $r \geqq 1$ then $q^{r} \geqq 3$ which is contrary to what we have just shown. Thus $x \in F$. Since $x$ was an arbitrary element of $G_{\phi}^{(k-1)}$ we may conclude that $G_{\phi}^{(k-1)} \leqq F$.

The final step in the proof is to prove that $\omega$ centralises $G / F_{2}(G)$. This will yield the theorem for since $G_{A}=G_{\omega}$ and $G_{\omega}^{(m-1)} \leqq F$ it follows that $G^{(m-1)} \leqq F_{2}(G)$. But if $1 \neq \omega^{r}$ then $\omega^{r}$ acts fixed-point-free on $F_{2}(G) / F$. Thus if $p$ is any prime dividing the order of $\omega, F_{2}(G) / F$ has derived length at most $l(p)$. Combining these two results we find that $G^{(t)} \leqq F$ contrary to the assumption that we are dealing with a counterexample.

Thus it remains to prove that $\omega$ centralises $G / F_{2}(G)$. The proof is in two steps. We first prove that if $\phi$ is an element of $A$ with prime order then $G_{\phi}$ covers $G / F_{2}(G)$. The second part of the proof is to show that for the same $\phi$ if $x \in G_{\phi}$ then $x \in F G_{\omega}$. From these two results we conclude that $G_{\omega}$ also covers $G / F_{2}(G)$ and hence that $\omega$ centralises $G / F_{2}(G)$. Suppose in the remainder of the proof that $a=|A|$ and that $\phi$ is an arbitrary, but fixed, element of $A$ of prime order.

Since $G$ is soluble we have $C_{G / F_{2}(G)}\left(F_{3}(G) / F_{2}(G)\right) \leqq F_{3}(G) / F_{2}(G)$. Applying this result to cosets of the form $x^{-1} x^{\phi} F_{2}(G)$ we see that in order to prove that $\phi$ centralises $G / F_{2}(G)$ it is sufficient to show that $\phi$ centralises $F_{3}(G) / F_{2}(G)$. Write $L=F_{3}(G)$. We aim to prove that $\phi$ centralises $L / F_{2}(G)$. Let $\pi$ denote the set of prime divisors of $\left|F_{2}(G): F\right|$. For each $r \in \pi$ let $P_{r}$ denote the Sylow $r$-subgroup of $F_{2}(G) / F$. Then $P_{r} / \Phi\left(P_{r}\right)$ may be con- 
sidered as an $L\{\phi\} / F_{2}(G)$-module over the Galois field of $r$ elements. Let $K_{\tau} / F_{2}(G)$ denote the kernel of the corresponding representation of $L\{\phi\} / F_{2}(G)$. Since $C_{G / F}\left(F_{2}(G) / F\right) \leqq F_{2}(G) / F$ we have $\bigcap_{r \in \pi} K_{r}=F_{2}(G)$. Therefore to prove that $\phi$ centralises $G / F_{2}(G)$ it is sufficient to show that for each $r \in \pi\left(L / K_{r}\right)_{\phi}=L / K_{r}$. Since $\phi$ acts fixed-point-free on $P_{r} / \Phi\left(P_{r}\right)$ for each $r \in \pi$, this will follow from [7], Theorem 3.1 if we can show that $L / F_{2}(G)$ is an $r^{\prime}$-group for each $r \in \pi$. (The exceptional cases either do not arise or are excluded by our hypotheses). We already know that $F$ is a $q$-group and hence that $F_{2}(G) / F$ is a $q^{\prime}$-group. We now prove that $L / F_{2}(G)$ is a $q$-group. Let $H / F$ denote the Hall $q^{\prime}$-subgroup of $L / F$. Since $L / F_{2}(G)$ is nilpotent and $F_{2}(G) / F$ is a $q^{\prime}$-group, $H$ is characteristic in $L$. But $L$ is a characteristic subgroup of $G$ and hence $H$ is characteristic in $G$. Since $G_{\phi}$ is a $q$-group $\phi$ acts fixed-point-free on $H / F$. Since $\phi$ is of prime order we deduce that $H / F$ is nilpotent and hence that $H=F_{2}(G)$. This completes the proof that $\phi$ centralises $G / F_{2}(G)$.

We now conclude the proof by showing that if $x \in G_{\phi}$ then $x \in G_{\omega} F$. Let $x \in G_{\phi}$. Since $G_{\omega}=G_{A}$ is normal in $G_{\phi} x$ normalises $G_{\omega}$. Let $w \in F_{i}$ for an arbitrary but fixed $i$. Using the additive notation again we have

so that

$$
w\left(1+\omega+\omega^{2}+\cdots+\omega^{a-1}\right) \in F_{\omega}
$$

Therefore

$$
w\left(1+\omega+\omega^{2}+\cdots+\omega^{a-1}\right) x \in F_{\omega} .
$$

$$
w\left(1+\omega+\omega^{2}+\cdots+\omega^{a-1}\right) x=\left(w\left(1+\omega+\omega^{2}+\cdots+\omega^{a-1}\right) x\right) \omega .
$$

Equating the $F_{i}$ components of both sides we have:

$$
w x=w x^{\omega} .
$$

But $w$ was an arbitrary element of $F_{i}$ and $F$ is the sum of the $F_{i}$ so we have $v x=v x^{\omega}$ for all $v \in F$. Therefore $x^{\omega} x^{-1} \in C_{G}(F)=F$. Hence $x^{\omega} \in F x$ from which we may conclude the desired result.

This completes the proof of Theorem 1 .

\section{Proof of Theorem 2}

We use the same methods and notation which are set out in the first paragraph of the proof of Theorem 1. For the same reasons as are set out in the second paragraph of the proof of Theorem 1 we know that $F=F(G)$ is the unique minimal normal $A$-subgroup of $G$. Thus $F$ is an elementary abelian $q$-group where $q$ is some prime.

Let $\omega \in A^{\not \#}$ and suppose that $G / F=(G / F)_{\omega}$. Then, since $G_{\omega}$ is nilpotent, $G / F$ is nilpotent. The minimal nature of $G$ now implies that $G / F$ 
is an $r$-group for some prime $r$. Since $G$ is a $p^{\prime}$-group, we may choose an $A$ invariant Sylow $r$-subgroup $R$ to complement $F$ in $G$. Since $(G / F)_{\omega}=G / F$, we have $R_{\omega}=R$. Now $Z(G)=1$ for otherwise $F \leqq Z(G)$ which is false. Since $G_{\omega}$ is nilpotent and $F$ is abelian we have $F_{\omega} \leqq C_{G}(F R)=Z(G)=1$. Therefore $F_{\omega}=1$. Now consider the join of the subgroups $F_{\phi}$ where

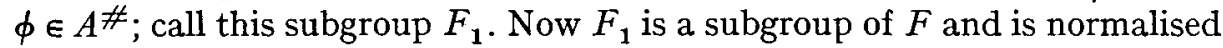
by $A$. If $F_{1} \neq F$ then by Maschke's theorem there exists a subgroup $F_{2}$ complementary to $F_{1}$ in $F$ and normalised by $A$. By the definition of $F_{1}$, each element of $A^{\#}$ must act fixed-point-free on $F_{2}$, which contradicts lemma 2 since $A$ is not cyclic. Therefore $F_{1}=F$. Now suppose that $\phi \in A$ but $\phi \notin\{\omega\}$. Since $R_{\omega}=R$ and $A=\{\omega, \phi\}$, we have $R_{\phi}=R_{A}$. But $R_{A}$ is an $r$-group and $F$ is an $r^{\prime}$-group so that $R_{A}$ centralises $F_{\theta}$ for each $\theta \in A^{\not \#}$. Since $F$ is the join of the $F_{\theta}$ for $\theta \in A^{\not \#}$, we can conclude that $R_{\phi}=R_{A}$ centralises $F$. But since $G$ is soluble, this implies that

$$
R_{\phi} \leqq R \cap C_{G}(F) \leqq R \cap F=1 .
$$

Therefore $\phi$ induces a fixed-point-free automorphism of order $p$ on $R$. Since $R \cong G / F$ it follows that $G / F \in V$ and hence that $G$ satisfies the conclusion of Theorem 2. Thus we may assume that for no $\omega \in A^{\#}$ is $(G / F)_{\omega}=G / F$.

We now consider $F$ as a $G F(q)(N)$-module where $N=G A / F$. By extending the field to a splitting field $\mathscr{F}$ of all subgroups of $N$ we may obtain, as in [6], page 118, an $\mathscr{F}(N)$-module $V$ with the following properties:

(1) $V$ is an irreducible and faithful $\mathscr{F}(N)$-module;

(2) $V_{\omega}$ is centralised by any $q^{\prime}$-element of $M_{\omega}$ where $\omega$ is any element of $A^{\#}$ and $M$ denotes the group $G / F$.

As an $\mathscr{F}(M)$-module, by Clifford's theory, $V=W_{1} \oplus W_{2} \oplus \cdots \oplus W_{s}$ where the $W_{i}$ are homogeneous components. Since $V$ is irreducible as an $\mathscr{F}(N)$-module, $A$ permutes the $W_{i}$ transitively. Let $\bar{A}=\left\{\omega \in A \mid W_{i} \omega=W_{i}\right\}$. Then, since $A$ is abelian, $A$ is independent of $i$. Also we have $s=|A: \bar{A}|$.

Assume that $s>1$ (so that $A \neq \bar{A}$ ). Let $\omega \in A-\bar{A}$. Let $w \in W_{i}$ for some fixed $i$. Then if

$$
u=w\left(1+\omega+\omega^{2}+\cdots+\omega^{p-1}\right)
$$

we have $u \in V_{\omega}$. Now if $x \in(F(M))_{\omega}$ then by (2), since $F(M)$ is a $q^{\prime}$-group, it follows that $u x=u$. Equating the $W_{i}$-components of both sides of this relation we find that $w x=w$. Therefore $x$ is represented trivially on $W_{i}$. Since this is true for all $i, x$ is represented trivially on the sum $V$ of the $W_{i}$. Hence by (1), we have $x=1$. Thus $(F(M))_{\omega}=1$. Now let $\theta \in \bar{A}$ and suppose that $(F(M))_{\theta} \neq F(M)$. Then there exists an abelian $A$-factor, $T$, of $F(M)$ such that $T_{\theta} \neq T$. Since $A$ is abelian, $T_{\theta}$ is normalised by $A$. 
Thus $A$ operates on $T / T_{\theta}$ in such a way that each element of $A^{\#}$ has a trivial centraliser in $T / T_{\theta}$. But since $A$ is not cyclic, this is contrary to Lemma 2. Therefore, if $\theta \in \bar{A}$ then we have $(F(M))_{\theta}=F(M)$. Since $C_{M}(F(M)) \leqq F(M)$, it follows that $M_{\theta}=M$. However this is contrary to the assumption made at the end of the second paragraph of the proof. Thus we conclude that $s=1$ and $A=A$. In other terms what we have shown is that $V$ is a homogeneous $\mathscr{F}(M)$-module.

We now show that if $\omega \in A^{\#}$ and $L$ is a non-trivial normal $A$-subgroup of $M$ then $L_{\omega} \neq 1$. It is sufficient to consider the case in which $L$ is abelian. Considering $V$ as an $\mathscr{F}(L)$-module we have $V=W_{1} \oplus W_{2} \oplus \cdots \oplus W_{r}$ where the $W_{i}$ are the homogeneous components. For at least one $i$ we have $W_{i} \omega=W_{i}$; we suppose that this is the case for $i=1$. For each $i$, the representation of $L$ on $W_{i}$ is defined by some character $\chi_{i}$. Since the characters $\chi_{i}$ are all conjugate in $M$ and $L$ is represented faithfully on $V$, none of the characters $\chi_{i}$ can be the trivial character. We show that $L_{\omega} \neq 1$ by showing that if $L_{\omega}=1$ then $\chi_{1}$ is the trivial character. Suppose then that $L_{\omega}=1$ and for convenience we write $\chi=\chi_{1}$. Now if $x \in L$ and $w \in W_{1}$ then $w \omega \in W_{1}$ and we have:

$$
\chi(x) w=w x=w \omega\left(\omega^{-1} x \omega\right) \omega^{-1}=w \omega\left(x^{\omega}\right) \omega^{-1}=\chi\left(x^{\omega}\right)(w \omega) \omega^{-1}=\chi\left(x^{\omega}\right) w .
$$

Thus for all $x \in L$ it follows that $\chi\left(x^{\omega}\right)=\chi(x)$. Now since $L_{\omega}=1$, we have $x x^{\omega} x^{\omega^{2}} \cdots x^{\omega^{p-1}}=1$. Therefore

$$
1=\chi(1)=\chi\left(x x^{\omega} x^{\omega^{2}} \cdots x^{\omega^{p-1}}\right)=(\chi(x))^{p} .
$$

But if $x$ is of order $n$ then the greatest common divisor of $p$ and $n$ is 1 and $(\chi(x))^{n}=1$. Hence $\chi(x)=1$, showing that $\chi$ is the trivial character. This proves that $L_{\omega} \neq 1$ for any $\omega \in A^{\# \text {. }}$

By Lemma 2 we know that for some $\omega \in A^{\# \#}$ we have $F_{\omega} \neq 1$. We now choose and fix $\omega \in A$ such that $F_{\omega} \neq 1$. Now by our method for obtaining the module $V$ it follows that $V_{\omega} \neq 1$. We now show that if $L$ is a non-trivial normal $\omega$-subgroup of $M$ then $L_{\omega} \neq L$. Since $M$ is soluble we may suppose that $L \leqq F(M)$. Now if $L_{\omega}=L$ then, by (2) since $L$ is a $q^{\prime}$-group, $L$ centralises $V_{\omega}$. But as an $L$-module $V$ is a sum of conjugate homogeneous components. Therefore since $L$ centralises $V_{\omega}, L$ must be represented trivially on $V$. But then $L=1$ which is contrary to our hypothesis.

Keeping $\omega$ as in the last paragraph and applying the conclusion of that paragraph we deduce that $(Z(M))_{\omega}=1$. Therefore $Z(M)=1$ and $M$ is not nilpotent. By the minimality of $G, M / F(M)$ must be irreducible under $A$. Thus $M / F(M)$ is an elementary abelian $s$-group for some prime $s$. Let $S$ denote a Sylow $s$-subgroup of $M$ normalised by $A$ and suppose that $S \cap F(M) \neq 1$. Then $Z(S) \cap F(M) \neq 1$ since $S \cap F(M)$ is normal in $S$. But $Z(S) \cap F(M) \leqq Z(M)$ since $Z(S) \cap F(M)$ is centralised by $S$ and all 
the $s^{\prime}$-elements of $M$ since they lie in $F(M)$. Thus $S \cap F(M)=1$ and $S$ is a complement of $F(M)$ in $M$.

Since $A$ acts irreducibly on $S$ we have $S_{\theta}=S$ for some $\theta \in A$. Now $1 \neq(F(M))_{\theta}$ is centralised by $S$ since $M_{\theta}$ is nilpotent and $F(M)$ is an $s^{\prime}$-group. But then $(Z(F(M)))_{\theta} \leqq Z(M)=1$, a contradiction. This completes the proof of Theorem 2 .

\section{Examples}

Before proceeding to the constructions of the promised examples, we make two remarks which will be useful.

REMARK 1. Let $H$ and $K$ be soluble groups with derived lengths $m$ and $n$ respectively. If the orders of $H$ and $K$ are relatively prime then the derived length of the wreath product, $H$ wr $K$, is precisely $m+n$.

Proof. Even without the assumption on the orders, the derived length of $H$ vor $K$ is not greater than $m+n$. Let $N$ denote the normal subgroup of $H \operatorname{wr} K$ which is isomorphic to the direct product of $|K|$ copies of $H$. Identify $K$ with a complementary subgroup of $N$ in $H$ wr $K$. Now it is easily checked that $\left(N, K^{(n-1)}\right)$ has derived length $m$. Since the orders of $H$ and $K$ are relatively prime, $\left(N, K^{(n-1)}\right)$ is the first term of the lower Fitting series of $N\left(K^{(n-1)}\right)$. Therefore $\left(N, K^{(n-1)}\right)=\left(\left(N, K^{(n-1)}\right), K^{(n-1)}\right)$. This means that $\left(N, K^{(n-1)}\right) \leqq(H \text { wr } K)^{(n)}$ and hence that $H$ wr $K$ has derived length at least $m+n$.

REMARK 2. Let $H$ and $K$ be finite groups and let $\bar{A}$ be a group of operators on $H$. Then there exists a group of operators $A$ isomorphic to $\bar{A}$ on the group $H \operatorname{wr} K$.

Proof. Let $A$ be a group isomorphic to $\bar{A}$. Let $N$ and $K$ denote the same subgroups of $H$ wr $K$ as in the proof of Remark 1. The action of each element of $A$ on $H$ wr $K$ is now defined: if $\omega \in A$ we let $\omega$ centralise $K$ and operate on each of the direct factors of $N$ which are isomorphic to $H$, as the corresponding element of $A$ acts on $H$. It is easily checked that $\omega$ is an operator on $H$ wr $K$.

Example 1. Let $H$ be a group with a fixed-point-free automorphism $\bar{\omega}$ of order $p$, and derived length $l(p)$. We may assume that $H$ is an $r$-group where $r$ is a prime. Let $\bar{A}=\{\bar{\omega}\}$ and let $K$ be an arbitrary $q$-group where $q$ is some prime not dividing $2 r p$. Now form $H$ wr $K$ and its group of automorphisms, $A$, as described in Remark 2. Let $L$ denote the extension of $H$ wr $K$ by the automorphism group $A$. Denote by $\Gamma$ the group $C_{a}$ wr $L$ where $C_{q}$ is the cyclic group of order $q$. $\Gamma$ contains a normal subgroup $G$ 
of index $p$. Let $\omega$ be the automorphism of $G$ induced by an element of $\Gamma$ of order $p$.

Now $G$ has a series, $G>N_{1}>N_{2}>1$, of normal $\omega$-subgroups. The factor group $G / N_{1}$ is isomorphic to $K$ and is centralised by $\omega$. The other factor, $N_{1} / N_{2}$, is isomorphic to the direct product of $|K|$ copies of $H$, and is acted on fixed-point-free by $\omega$. Finally $N_{2}$ is an elementary abelian $q$-group and is the Fitting subgroup of $G$.

By our choice of $H$, the derived length of $H$ is $l(p)$. Let $d$ and $n$ denote the derived lengths of $K$ and $G_{\omega}$ respectively. Since $G / N_{2}$ is isomorphic to $H$ wr $K$ and $N_{2}$ is the Fitting subgroup of $G$, the derived length of $G / F(G)$ is, by Remark $1, l(p)+d$. Since $G_{\omega}$ is a $q$-group and $G_{\omega} / N_{2} \cap G_{\omega}$ is isomorphic to $K$ it follows that $n$ is either $d$ or $d+1$. Now, provided that the hypotheses of Theorem 1 are satisfied by $G$ and the automorphism group $\{\omega\}, G^{(t)}$ is nilpotent where $t=l(p)+n-1$. Thus we have $l(p)+n-1 \geqq l(p)+d$, so that $n$ is at least $d+1$. It follows that $n=d+1$ and that $G^{(t-1)}$ is not nilpotent.

The hypotheses of Theorem 1 will be satisfied if $r \neq 2$. This can certainly be achieved if the definition of $l(p)$ has been changed as mentioned in the discussion of the results.

EXAMPLE 2. Choose a pair of primes $(p, q)$ and a group $H$ so as to satisfy the following conditions:

(a) $l(p) \geqq l(q)$;

(b) there exists a fixed-point-free automorphism $\bar{\omega}$ of order $p$, acting on the group $\bar{H}$;

(c) the derived length of $\bar{H}$ is $l(p)$; and

(d) $q$ does not divide the order of $\bar{H}$.

Let $r$ denote some prime, distinct from both $p$ and $q$, and not dividing $|\bar{H}|$, such that there exists a solution $\alpha,(\alpha \neq 1)$, of the equation $x^{q} \equiv 1$ $(\bmod r)$. Form the group $\bar{H}\{\omega\}$ by adjoining $\bar{\omega}$ to $\bar{H}$ and let ' $\Gamma$ ' denote the group $C_{r}$ wr $\bar{H}\{\bar{\omega}\}$. If $G$ denotes the normal subgroup of $\Gamma$ of index $p$ then there is induced on $G$ an automorphism $\omega$ of order $p$ by an element of order $p$ in $\Gamma$. Since $p$ does not divide $|G|, \omega$ normalises some Hall $r^{\prime}$-subgroup $H$ of $G$. Clearly $H$ is isomorphic with $\bar{H}$. Now $F=F(G)$ is an elementary abelian $r$-group and we have $G=H F$. From the construction it follows that $H_{\omega}=1$ so that $G_{\omega}=F_{\omega}$ is abelian.

We now define a second automorphism $\phi$ of $G$. If $x \in H$ and $y \in F$ then we set $(x y)^{\phi}=x\left(y^{\alpha}\right)$. It is routine to check that $\phi$ is an automorphism of $G$ of order $q$ and that $\phi$ commutes with $\omega$. Since we have $\alpha \neq 1$ it follows that $G_{\phi}=H$.

Now we define $A=\{\omega, \phi\}$. Then $G_{A}=G_{\omega} \cap G_{\phi}=1$. Now $G / F \cong H$ so that the derived length of $G / F$ is $l(p)$. If $t, n$ and $m$ are defined as in 
Theorem 1 then we have $t=n=l(p)$ and $m=0 . G^{(t-1)}$ is not nilpotent and $l(q)+m-1 \leqq l(p)+m-1<t$.

EXAmpLE 3. Let $H$ be a group of derived length 3 with a fixed-point-free automorphism $\omega$ of order four. (An example of such a group is given in [5] page 294; although this example has a nonabelian Sylow 3-subgroup, a similar construction will yield such groups with abelian Sylow 3-subgroups). In order to ensure that the group which we construct will satisfy the hypotheses of Theorem 1, we suppose that $H$ has an abelian Sylow 3-subgroup.

Choose an odd prime $r$ which does not divide $|H|$. Form the group $\Gamma=C_{r}$ wr $H\{\omega\}$ where $H\{\omega\}$ is the group obtained by adjoining $\omega$ to $H$. $\Gamma$ has a normal subgroup $G$ of index 4 . The factor group $G / F(G)$ is isomorphic with $H$ and $F(G)$ is an elementary abelian $r$-group. An element of order 4 in $\Gamma$ induces an automorphism $\phi$ of $G$ with $(G / F(G))_{\phi}=1$. Thus $G_{\phi} \leqq F(G)$ is abelian. Since $\phi$ induces a fixed-point-free automorphism of order 2 on $(G / F(G))_{\left(\phi^{2}\right)}$, this group is abelian. Thus $G_{\left(\phi^{2}\right)}$ has derived length at most 2 .

If Theorem 1 were to apply to the group $G$ with the automorphism group $A=\{\phi\}$, then since $l(2)=1$, we would have $G^{\prime \prime}$ nilpotent. This is clearly false, the missing hypothesis being that $G_{A}=G_{\phi}$ is not normal in $G_{\left(\phi^{2}\right)}$.

\section{References}

[1] W. Burnside, Theory of Groups of Finite Order (New York, 2nd ed. 1911; Dover, 1955).

[2] P. Hall and G. Higman, 'On the $p$-length of $p$-soluble groups and reduction theorems for Burnside's problem', Proc. London Math. Soc. (3) 6 (1956), 1-42.

[3] G. Higman, 'On Groups and Rings which possess Automorphisms without Non-trivial Fixed Elements', J. London Math. Soc. 32 (1957), 321-334.

[4] Hans Kurzweil, 'Auflosbare Gruppen, die eine abelsche Automorphismengruppe gestatten, deren Fixpunktgruppe nilpotent ist', J. Algebra 10 (1968), 92-101.

[5] L. G. Kovács, 'Groups with Regular Automorphisms of Order Four', Math. Zeit. 75 (1961), 277-294.

[6] L. G. Kovács and G. E. Wall, 'Involutory Automorphisms of Groups of Odd Order and their Fixed-point Groups', Nagoya Math. J. 27 (1966), 113-120.

[7] E. Shult, 'On Groups Admitting Fixed-point-free Abelian Operator Groups', Illinois J. Math. 9 (1965), 701-720.

[8] J. G. Thompson, 'Finite Groups with Fixed-point-free Automorphisms of Prime Order', Proc. Nat. Acad. Sci. U.S.A. 45 (1959), 578-581.

[9] J. N. Ward, 'Involutory Automorphisms of Groups of Odd Order', J. Austral. Math. Soc. $6(1966), 480-494$.

[10] D. Gorenstein and I. Hernstein, 'Finite Groups Admitting a Fixed-point-free Automorphism of Order 4', Amer. J. Math. 83 (1961), 71-78. 